Bull. Austral. Math. Soc.

VOL. 48 (1993) [393-406]

\title{
CHARACTERISATIONS OF QUASICONVEX FUNCTIONS
}

\section{Dinh The LuC}

In this paper we introduce the concept of quasimonotone maps and prove that a lower semicontinuous function on an infinite dimensional space is quasiconvex if and only if its generalised subdifferential or its directional derivative is quasimonotone.

\section{INTRODUCTION}

As far as we know quasiconvex functions were first mentioned by de Finetti in his work "Sulle Straficazioni Convesse" in 1949, [6]. Since then much effort has been focused on the study of this class of functions for they have much in common with convex functions and they are used in several areas of science including mathematics, operations research, economics et cetera (see [15]). One of the most important properties of convex functions is that their level sets are convex. This property is also a fundamental geometric characterisation of quasiconvex functions which sometimes is treated as their definition. However, the most attractive characterisations of quasiconvex functions are those which involve gradients (a detailed account of the current state of research on the topic can be found in [1]). As to generalised derivatives of quasiconvex functions, very few results exist (see $[1,7]$ ). In [7], a study of quasiconvex functions is presented via Clarke's subdifferential, but the authors restrict themselves to the case of Lipschitz functions on a finite dimensional space only.

The aim of our paper is to characterise lower semicontinuous quasiconvex functions in terms of generalised (Clarke-Rockafellar) subdifferentials and directional derivatives in infinite dimensional spaces. Namely, we shall first introduce a new concept of quasimonotone maps; and then we prove the equivalence between the quasiconvexity of a function and the quasimonotonicity of its generalised subdifferential and its directional derivative. A modified version of Zagrodny's approximate mean value theorem (Lemma 3.1) and another version of mean value theorem via directional derivatives (Lemma 5.1) are the main tools we use in establishing the results, thus avoiding the conventional condition on Lipschitz continuity or differentiability of the function considered.

\section{Received 1st February, 1992}

The paper was partly written when the author was at the University of Erlangen-Nurnberg under a grant of the Alexander von Humboldt Foundation.

The author would like to thank Prof. J. Jahn of the University of Erlangen-Nurnberg for useful discussions on the first draft of the paper.

Copyright Clearance Centre, Inc. Serial-fee code: 0004-9729/93 \$A2.00+0.00. 
The paper is structured as follows. In the next section we introduce the concept of quasimonotonicity for set valued maps from a vector space to its dual. We show that in the case of linear operators there is no difference between this concept and the monotonicity known in convex analysis. In Section 3 we prove that a function is quasiconvex if and only if its generalised subdifferential is quasimonotone. It is also pointed out that quasimonotone subdifferentials are generally not maximal. Section 4 is devoted to the monotonicity and quasimonotonicity of bifunctions and their relations to the corresponding notions introduced in Section 2. In Section 5 another characterisation of quasiconvex functions is given by means of directional derivatives. Here we also illustrate by two corollaries how to apply our results to derive the characterisations previously obtained by other authors.

\section{QUASIMONOTONE MAPS}

Let $X$ be a real topological vector space and $F$ a set valued map from $X$ to its topological dual $X^{\prime}$. The pairing between $X$ and $X^{\prime}$ is denoted by $\langle.,$.$\rangle .$

Definition 2.1: $F$ is said to be quasimonotone if for every $x, y \in X$ and $x^{*} \in$ $F(x), y^{*} \in F(y)$ one has

$$
\min \left\{\left\langle x^{*}, y-x\right\rangle,\left\langle y^{*}, x-y\right\rangle\right\} \leqslant 0 .
$$

We recall that $F$ is said to be monotone if for $x, y, x^{*}$ and $y^{*}$ as above,

$$
\left\langle x^{*}, y-x\right\rangle+\left\langle y^{*}, x-y\right\rangle \leqslant 0
$$

It is evident that any monotone map is quasimonotone, but the converse is not true in general. For instance, take $X=\mathbb{R}$ and

$$
F(x)= \begin{cases}-x & \text { if } x \leqslant 0 \\ 0 & \text { otherwise }\end{cases}
$$

Of course, the map $F$ is quasimonotone, but not monotone. The following case is a useful exception.

Proposition 2.2. Let $X$ be a real Hilbert space and $A$ a linear operator on $X$. Then the following conditions are equivalent:

(1) A is monotone;

(2) $A$ is quasimonotone;

(3) the symmetric part of $A$ is positive semidefinite. 
Proof: The implication $(1) \Rightarrow(2)$ is obvious. For the implication $(2) \Rightarrow(3)$ we suppose to the contrary that the symmetric part $A_{\&}=\left(A+A^{T}\right) / 2$, where $A^{T}$ is the transposition of $A$, is not positive semidefinite, that is, there exists a vector $x \in X$ such that $\langle x, A, x\rangle<0$. Taking $y=-x$ and observing that $\langle x, A x\rangle=\langle x, A, x\rangle$, we have

$$
\begin{aligned}
& \langle A x, y-x\rangle=-2\langle x, A x\rangle>0 \\
& \langle A y, x-y\rangle=-2\langle x, A x\rangle>0
\end{aligned}
$$

These inequalities show that $A$ is not quasimonotone. We show finally the implication $(3) \Rightarrow(1)$ (see [14] for the finite dimensional case). Suppose that $A$ is not monotone, that is, there exist two points $x, y \in X$ such that

$$
\langle A x, y-x\rangle+\langle A y, x-y\rangle>0
$$

Since $A=A_{a}+A_{a}$, where $A_{a}=\left(A-A^{T}\right) / 2$ is the antisymmetric part of $A$, the above inequality implies

$$
\left\langle A_{\mathfrak{s}}(x-y), y-x\right\rangle+\left\langle A_{a}(x-y), x-y\right\rangle>0
$$

or equivalently

$$
\langle A,(x-y), x-y\rangle<0 .
$$

This means that $A_{\mathbb{a}}$ is not positive semidefinite.

In [7] a definition of quasimonotone maps is also given. A discussion on the relation between the definition of [7] and that of our paper will be addressed in Penot and Quang's forthcoming paper [12].

\section{Subdifferentials of QuASICONVEX Functions}

Throughout this section $X$ will denote a Banach space and $f$ a lower semicontinuous function from $X$ to $\mathbb{R} \cup\{+\infty\}$. Assume that the value of the function is finite at a point $x \in X$. The generalised (Clarke-Rockafellar) subderivative of $f$ at $x$ in direction $v$ is defined by

$$
f^{\dagger}(x ; v):=\sup _{\varepsilon>0} \limsup _{(y, \alpha) \downarrow_{f} x ; t \downarrow 0} \inf _{u \in B(v, \varepsilon)} \frac{f(y+t u)-\alpha}{t}
$$

where $(y, \alpha) \downarrow_{f} x$ means that $y \rightarrow x, \alpha \rightarrow f(x), \alpha \geqslant f(y), B(v, \varepsilon)$ is the ball in $X$ with centre at $v$ and radius $\varepsilon$ (see $[3,13])$. The generalised subgradient of $f$ at $x$ is

$$
\partial f(x):=\left\{x^{*} \in X^{\prime}:\left\langle x^{*}, v\right\rangle \leqslant f^{\top}(x ; v) \text { for all } v \in X\right\}
$$


We recall also that $f$ is said to be quasiconvex if

$$
f(\lambda x+(1-\lambda) y) \leqslant \max \{f(x), f(y)\}
$$

for any $x, y \in X, \lambda \in[0,1]$. Our aim is to show that $f$ is quasiconvex if and only if $\partial f$ is quasimonotone. We need the following lemma which was established in [8] by using the method of [17]. For the convenience of the reader, a detailed proof is given below.

LEMma 3.1. Assume that $f(b)>f(a)$. Then there exists a sequence $\left\{x_{k}\right\} \subseteq X$ converging to some $x_{0} \in[a, b), x_{k}^{*} \in \partial f\left(x_{k}\right)$ such that for every $c=a+t(b-a)$ with $t \geqslant 1$ and for every $k$, one has $\left\langle x_{k}^{*}, c-x_{k}\right\rangle>0$.

Proof: Assume that $f(b)$ is finite. Following the method of [17], let us consider the functions $g_{k}, k=1,2, \ldots$ defined by

$$
g_{k}(x):=f(x)+\frac{f(b)-f(a)}{2\|b-a\|}\|x-b\|+k d_{[a, b]}(x),
$$

where $d_{[a, b]}(x)$ denotes the distance from $x$ to the interval $[a, b]$. Sometimes $d_{b}(x)$ is also used to denote $\|x-b\|$. Since $f$ is lower semicontinuous, the functions $g_{k}$ are lower semicontinuous too. In particular, they are bounded from below on some bounded closed neighborhood, say $B$ of $[a, b]$. By Ekeland's variational principle [3], for every fixed $k$, there exists a point $x_{k} \in B$ minimising the function $g_{k}+d_{x_{k}} / k$ over $B$. It is clear that $\lim _{k \rightarrow \infty} d_{[a, b]}\left(x_{k}\right)=0$. Hence, one may assume that $x_{k}$ is in the interior of $B$. Consequently, the subdifferential of the function $g_{k}+d_{x_{k}} / k$ at $x_{k}$ must contain the zero vector. Moreover, since the distance functions $d_{[a, b]}, d_{b}, d_{z_{k}}$ are convex Lipschitz, using the calculus rules for generalised subdifferential $[3,14]$, one has

$$
0 \in \partial f\left(x_{k}\right)+\frac{f(b)-f(a)}{2\|b-a\|} \partial d_{b}\left(x_{k}\right)+k \partial d_{[a, b]}\left(x_{k}\right)+\frac{1}{k} \partial d_{x_{k}}\left(x_{k}\right)
$$

or in other words, there exist

$$
x_{k}^{*} \in \partial f\left(x_{k}\right), u_{k}^{*} \in \partial d_{b}\left(x_{k}\right), v_{k}^{*} \in \partial d_{[a, b]}\left(x_{k}\right), w_{k}^{*} \in \partial d_{x_{k}}\left(x_{k}\right),
$$

such that

$$
x_{k}^{*}=-\frac{f(b)-f(a)}{2\|b-a\|} u_{k}^{*}-k v_{k}^{*}-\frac{1}{k} w_{k}^{*}
$$

As noticed above, $\lim _{k \rightarrow \infty} d_{[a, b]}\left(x_{k}\right)=0$. Furthermore, since $[a, b]$ is compact and $g_{k}(b)>$ $g_{k}(a)$, we may assume that $\lim _{k \rightarrow \infty} x_{k}=x_{0} \in[a, b], x_{0} \neq b$. There exists then a number $k_{1}$ such that

$$
\left\|x_{k}-x_{0}\right\|<\frac{1}{2}\left\|x_{0}-b\right\|, \quad \text { for } k>k_{1} .
$$


The aim now is to estimate $\left\langle x_{k}^{*}, c-x_{k}\right\rangle$. Denote by $b_{k} \in\left[c, x_{k}\right]$ a point minimising $d_{b}$ over $\left[c, x_{k}\right]$. It is clear that $\lim _{k \rightarrow \infty} b_{k}=b$. By this and (2), one may assume that $x_{k} \neq b_{k}$ and one can obtain the relation $\left\|b-b_{k}\right\| \leqslant d_{[a, b]}\left(x_{k}\right)$. Consequently,

$$
d_{[a, b]}\left(b_{k}\right) \leqslant d_{[a, b]}\left(x_{k}\right) \text {. }
$$

We express $c-x_{k}=\beta\left(b_{k}-x_{k}\right)$ for some $\beta>1$ depending on $k$ and calculate

$$
\left\langle u_{k}^{*}, c-x_{k}\right\rangle=\beta\left(\left\langle u_{k}^{*}, b_{k}-b\right\rangle+\left\langle u_{k}^{*}, b-x_{k}\right\rangle\right)
$$

Remembering that $u_{k}^{*} \in \partial d_{b}\left(x_{k}\right)$, we see that $\left\langle u_{k}^{*}, b-x_{k}\right\rangle=-\left\|x_{k}-b\right\|$. Moreover, since $\lim _{k \rightarrow \infty} b_{k}=b$ and $\lim _{k \rightarrow \infty}\left\|x_{k}-b\right\|=\left\|x_{0}-b\right\| \neq 0$, there exists an integer $k_{2}>k_{1}$ such that

$$
\left|\left\langle u_{k}^{*}, b_{k}-b\right\rangle\right|<\frac{1}{4}\left\|x_{k}-b\right\| \text { for } k>k_{2} .
$$

Hence (4) can be evaluated as

$$
\left\langle u_{k}^{*}, c-x_{k}\right\rangle \leqslant-\frac{3 \beta}{4}\left\|x_{k}-b\right\|, \quad \text { for } k>k_{2} .
$$

Furthermore, using (3) one has that

$$
\left\langle v_{k}^{*}, c-x_{k}\right\rangle=\beta\left\langle v_{k}^{*}, b_{k}-x_{k}\right\rangle \leqslant \beta\left(d_{[a, b]}\left(b_{k}\right)-d_{[a, b]}\left(x_{k}\right)\right) \leqslant 0 .
$$

Notice that $\left\|w_{k}^{*}\right\| \leqslant 1$, there can be found $k_{3}>k_{2}$ such that

$$
\frac{1}{k}\left\langle w_{k}^{*}, c-x_{k}\right\rangle<\frac{\beta}{8} \frac{f(b)-f(a)}{\|b-a\|}\left\|x_{k}-b\right\|, \quad \text { for } k>k_{3} .
$$

Combine the latter inequality with (1), (5) and (6) to obtain the estimate:

$$
\left\langle x_{k}^{*}, c-x_{k}\right\rangle \geqslant \frac{\beta}{4} \frac{f(b)-f(a)}{\|b-a\|}\left\|x_{k}-b\right\|>0,
$$

for every $k>k_{3}$. The sequence $\left\{x_{k}\right\}$ with $k>k_{3}$ will be such as required in the lemma. The case $f(b)=\infty$ can be manipulated as follows. Set

$$
\tilde{f}(x)= \begin{cases}f(x) & \text { if } x \neq b \\ f(a)+1 & \text { otherwise. }\end{cases}
$$

Then by the above proof the lemma is true for $\tilde{f}$. Since $\lim _{k \rightarrow \infty} x_{k}=x_{0} \neq b$, we may assume that $x_{k} \neq b$ for all $k$. At these points $\partial f$ and $\partial \tilde{f}$ coincide. Hence the lemma is also true for $f$. 
THEOREM 3.2. $f$ is quasiconvex if and only if $\partial f$ is quasimonotone.

Proof: Assume first that $f$ is quasiconvex. Let $x$ and $y$ be two arbitrary points in $X$ with $\partial f(x)$ and $\partial f(y)$ nonempty. If we can show that

$$
\min \left\{f^{\dagger}(x, y-x), f^{\dagger}(y, x-y)\right\} \leqslant 0,
$$

then the quasimonotonicity of $\partial f$ follows. Suppose to the contrary that the above relation does not hold, that is,

$$
f^{\dagger}(x, y-x)>0, \quad \text { and } \quad f^{\dagger}(y, x-y)>0
$$

By the definition of the generalised subderivative, there exist a positive $\varepsilon$, two sequences $\left\{x_{n}\right\},\left\{y_{n}\right\}$ converging to $x$ and $y$ respectively, two sequences of integers $\left\{t_{n}\right\},\left\{s_{n}\right\}$ decreasing to 0 such that

$$
\begin{aligned}
& \inf _{u \in B(y-x, e)} \frac{f\left(x_{n}+t_{n} u\right)-f\left(y_{n}\right)}{t_{n}}>0 \\
& \inf _{v \in B(x-y, \varepsilon)} \frac{f\left(y_{n}+s_{n} v\right)-f\left(y_{n}\right)}{s_{n}}>0 .
\end{aligned}
$$

Take $n$ sufficiently large so that

$$
\left\|x_{n}-x\right\|<\frac{1}{2} \varepsilon, \quad \text { and } \quad\left\|y_{n}-y\right\|<\frac{1}{2} \varepsilon .
$$

Then it is evident that $x_{n}-y_{n} \in B(x-y, \varepsilon), y_{n}-x_{n} \in B(y-x, \varepsilon)$. Replacing these vectors instead of $v$ and $u$ in (7) and (8) we have

$$
\begin{aligned}
& f\left(x_{n}+t_{n}\left(y_{n}-x_{n}\right)\right)-f\left(x_{n}\right)>0, \\
& f\left(y_{n}+s_{n}\left(x_{n}-y_{n}\right)\right)-f\left(y_{n}\right)>0 .
\end{aligned}
$$

Since $f$ is quasiconvex and for $n$ large, $t_{n}$ and $s_{n}$ are smaller than one, we have also

$$
\begin{aligned}
& f\left(x_{n}+t_{n}\left(y_{n}-x_{n}\right)\right) \leqslant \max \left(f\left(x_{n}\right), f\left(y_{n}\right)\right), \\
& f\left(y_{n}+s_{n}\left(x_{n}-y_{n}\right)\right) \leqslant \max \left(f\left(x_{n}\right), f\left(y_{n}\right)\right) .
\end{aligned}
$$

These inequalities contradict the system (9), (10).

For the converse part suppose to the contrary that $f$ is not quasiconvex, that is, there exist three points $a, b, c \in X$ with $b \in(a, c)$ such that $f(b)>\max \{f(a) ; f(c)\}$. Let us apply Lemma 3.1 to the function $f$ on $[a, b]$. There exists a sequence $\left\{x_{i}\right\}$ 
converging to $x_{0} \in[a, b), x_{i}^{*} \in \partial f\left(x_{i}\right)$ and for every $i$, one can find a positive $\varepsilon$ such that

$$
\left\langle x_{i}^{*}, y-x_{i}\right\rangle>0,
$$

for every $y \in X$ with $\|y-c\|<\varepsilon$. Take a point $b_{i} \in\left[c, x_{i}\right]$ with $\left\|b-b_{i}\right\|=d_{\left[c, x_{i}\right]}(b)$. It is evident that $\lim _{i \rightarrow \infty} b_{i}=b$ and $b_{i} \neq x_{i}$ when $i$ is large, say $i>i_{1}$ for some positive integer $i_{1}$. Moreover, by the lower semicontinuity of $f$, there exists $i_{2}>i_{1}$ such that $f\left(b_{i}\right)>f(c)$ for all $i>i_{2}$. Fix an index $i>i_{2}$ and apply Lemma 3.1 to the function $f$ on the interval $\left[c, b_{i}\right]$. There exists a sequence $x_{i, k}^{*} \in \partial f\left(x_{i, k}\right)$ such that

$$
\left\langle x_{i, k}^{*}, x_{i}-x_{i, k}\right\rangle>0 \text {. }
$$

(Note that $x_{i}=c+t\left(b_{i}-c\right)$ for some $t>1$ because $b_{i} \in\left[c, x_{i}\right]$ and $b_{i} \neq x_{i}, b_{i} \neq c$ ). Furthermore, since $\lim _{k \rightarrow \infty} x_{i, k}=x_{i, 0} \in\left[c, b_{i}\right]$, one sees that $\lim _{k \rightarrow \infty} c_{i, k}=c$, where

$$
c_{i, k}=x_{i}+\frac{\left\|c-x_{i}\right\|}{\left\|x_{i, k}-x_{i}\right\|}\left(x_{i, k}-x_{i}\right) .
$$

Hence for the given $\varepsilon$, there exists an integer $k_{1}$ (depending on $i$ ) such that $\left\|c_{i, k}-c\right\|<$ $\varepsilon$, for $k>k_{1}$. Consequently, if we take an index $i>i_{2}$ and for this fixed $i$, another index $k>k_{1}$, then by (11),

$$
\left\langle x_{i}^{*}, c_{i, k}-x_{i}\right\rangle=\frac{\left\|c-x_{i}\right\|}{\left\|x_{i, k}-x_{i}\right\|}\left\langle x_{i}^{*}, x_{i, k}-x_{i}\right\rangle>0 .
$$

This and (12) show that $\partial f$ is not quasimonotone. The proof is complete.

Corollary 3.3. Let $A$ be a symmetric $n \times n$ matrix. Then the following properties of the quadratic form $f(x)=x A x$ are equivalent:

(i) $f$ is convex;

(ii) $f$ is quasiconvex;

(iii) $A$ is positive semidefinite.

ProOF: By Proposition 2.2 and Theorem 3.2.

Other corollaries will be given in Section 5. Let us now mention that the subdifferential of a lower semicontinuous convex function $f$ is maximal monotone (see $[8,14])$ in the sense that there exists no monotone map $A$ from $X$ to $X^{\prime}$ such that $A \neq \partial f$ and the graph of $\partial f$ is contained in the graph of $A$. This fact is no longer true for quasiconvex functions. For instance, the function $f$ on $\mathbb{R}$ defined by

$$
f(x)= \begin{cases}-(-x)^{1 / 2} & \text { if } x \leqslant 0 \\ x^{1 / 2} & \text { otherwise }\end{cases}
$$

is quasiconvex, hence $\partial f$ is quasimonotone. Nevertheless the map $A$ whose graph is obtained by adding the point $(0,0)$ to the graph of $\partial f$ is quasimonotone and different from $\partial f$. 


\section{Quasimonotone Bifunctions}

Let $X$ be a real vector space and $h$ a function from the product space $X \times X$ to $\mathbb{R} \cup\{ \pm \infty\}$. To avoid the confusion between the concept of quasimonotonicity in the second section and the one defined now, we shall call $h$ a bifunction on $X$.

DEFINITION 4.1: $h$ is said to be monotone (respectively, quasimonotone) if for every $x, y \in X$,

$$
\begin{gathered}
h(x, y-x)+h(y, x-y) \leqslant 0, \\
(\text { respectively } \min \{h(x, y-x), h(y, x-y)\} \leqslant 0) .
\end{gathered}
$$

It is clear that every monotone bifunction is quasimonotone, but not vice versa. Similarly to Proposition 1.2, an exception can be expected for the linear case. Let $A$ be a linear bifunction on $X$. We define the symmetric and antisymmetric parts of $A$ as follows

$$
\begin{aligned}
& A_{s}(x, y)=\frac{1}{2}(A(x, y)+A(y, x)) \\
& A_{\mathbf{a}}(x, y)=\frac{1}{2}(A(x, y)-A(y, x)) .
\end{aligned}
$$

Proposition 4.2. For the linear bifunction $A$ on $X$, the following properties are equivalent:

(i) $A$ is monotone;

(ii) $A$ is quasimonotone ;

(iii) $A_{\text {a }}$ is positive semidefinite.

Proof: The proof is similar to Proposition 2.2, so we omit it.

Let $f$ be, as in the previous section, a lower semicontinuous function from a Banach space $X$ to $\mathbb{R} \cup\{+\infty\}$. The generalised subderivative $f^{\dagger}$ can be regarded as a bifunction on $X$. Below we give a link between the monotonicity (respectively, quasimonotonicity) of the bifunction $f^{\dagger}$ and that of the subdifferential $\partial f$.

Proposition 4.3. The bifunction $f^{\dagger}$ is monotone (respectively, quasimonotone) if and only if the subdifferential map $\partial f$ is monotone (respectively, quasimonotone).

Proof: The "only if" part is straighforward from the definitions of subdifferential and Definitions 2.1, 4.1. For the "if" part, assume that $\partial f$ is monotone. By Theorem 3.1 of $[8], f$ is a convex function. Hence its generalised derivatives coincide with its directional upper derivatives (see the definition in the next section, or [9]), which by Proposition 3.2 of [9] is monotone in the sense of Definition 4.1. Now assume that $\partial f$ 
is quasimonotone. By Theorem 3.2 the function is quasiconvex. The proof of Theorem 3.2 also reveals that for any $x, y \in X$ where $f(x)$ and $f(y)$ are finite,

$$
\min \left\{f^{\dagger}(x, y-x), f^{\dagger}(y, x-y)\right\} \leqslant 0
$$

Hence the bifunction $f^{\dagger}$ is quasimonotone.

\section{Directional Derivatives}

Let $X$ be a real topological vector space and $f$ a lower semicontinuous function from $X$ to $\mathbb{R} \bigcup\{+\infty\}$. We recall that the directional upper and lower derivatives of $f$ at $x \in X$, where $f(x)$ is finite, are the maps $f_{+}^{\prime}(x, v)$ and $f_{-}^{\prime}(x, v)$ from $X$ to $\mathbb{R} \bigcup\{ \pm \infty\}$ defined by

and

$$
\begin{aligned}
& f_{+}^{\prime}(x, v)=\limsup _{t \downarrow 0} \frac{f(x+t v)-f(x)}{t} \\
& f_{-}^{\prime}(x, v)=\liminf _{t \downarrow 0} \frac{f(x+t v)-f(x)}{t}
\end{aligned}
$$

for every $v \in X$. Whenever these two limits are equal, the common limit is called the directional derivative of $f$ at $x$ in direction $v$ and denoted by $f^{\prime}(x, v)$. We shall adopt the convention $f^{\prime}(x, v)=-\infty$ for every $v \in X$ if $f(x)=+\infty$. The following result is a version of the mean value theorem.

LEммA 5.1. Let $a, b \in X, a \neq b$, where $f(a)$ is finite. Let $f_{0}$ denote $f(b)-f(a)$ if $f(b)$ is finite, or any fixed positive number if $f(b)=+\infty$. Then there exists a point $c \in[a, b)$ such that $f_{-}^{\prime}(c, b-a) \geqslant f_{0}$.

Proof: We consider first the case $f(a)=f(b)$. Since $f$ is lower semicontinuous, there exists a point $c \in[a, b]$ minimising $f$ over $[a, b]$. One may assume $c \in[a, b)$ because $f(a)=f(b)$. For $t$ small enough, $c+t(b-a) \in[a, b]$, hence $f(c+t(b-a)) \geqslant$ $f(c)$. It is now evident that $f_{-}^{\prime}(x, b-a) \geqslant 0$. Assume now that $f(b) \neq f(a)$. If $f(b)$ is infinite, and given a positive $f_{0}$, define a function

$$
\tilde{f}(x):= \begin{cases}f(a)+f_{0} & \text { if } x=b \\ f(x) & \text { otherwise. }\end{cases}
$$

It is clear that if the lemma is true for $\tilde{f}$, then it is also true for $f$ because the point $c$ to be found is different from $b$. Thus, we may assume that $f(b)$ is finite. Consider the following function on $[0,1]$ :

$$
g(t):=f(a+t(b-a))-[f(a)+t(f(b)-f(a))]
$$


for $t \in[0,1]$. Since $f$ is lower semicontinuous, so must $g$ be. Moreover $g(0)=g(1)=0$. By the first part, there exists a point $t_{0} \in[0,1)$ such that $g_{-}^{\prime}\left(t_{0}, 1\right) \geqslant 0$. Let us calculate $g_{-}^{\prime}\left(t_{0}, 1\right)$. By the definition

$$
\begin{aligned}
g_{-}^{\prime}\left(t_{0}, 1\right) & =\liminf _{t \downarrow 0} \frac{g\left(t_{0}+t\right)-g\left(t_{0}\right)}{t} \\
& =\liminf _{t \downarrow 0} \frac{f(c+t(b-a))-f(c)-t(f(b)-f(a))}{t} \\
& =f_{-}^{\prime}(c, b-a)-(f(b)-f(a)),
\end{aligned}
$$

where $c=a+t_{0}(b-a)$. In this way, $f_{-}^{\prime}(c, b-a)=g_{-}^{\prime}\left(t_{0}, 1\right)+f(b)-f(a) \geqslant f_{0}$, and the proof is complete.

It is evident that Lemma 5.1 is also true for directional upper derivatives. This case has been used in [9] to prove that $f$ is convex if and only if $f_{+}^{\prime}$ is monotone. Using Lemma 5.1 instead of Proposition 2.2 of [9] one can improve the above result as follows: $f$ is convex if and only if $f_{-}^{\prime}$ is monotone. Now we proceed to the main result of this section.

THEOREM 5.2. The function $f$ is quasiconvex if and only if the bifunction $f_{-}^{\prime}$ is quasimonotone. The same is true for $f_{+}^{\prime}$.

Proof: Assume that $f$ is quasiconvex. For every two points $x, y \in X$, say $f(y) \geqslant$ $f(x)$, if $f(y)=\infty$, by the definition $f_{-}^{\prime}(y ; x-y)=-\infty$. If $f(y)$ is finite, by the quasiconvexity of $f, f(y+t(x-y)) \leqslant \max \{f(x), f(y)\}=f(y)$ for every $t \in[0,1]$. Hence $f_{-}^{\prime}(y, x-y) \leqslant 0$ and indeed $f_{-}^{\prime}$ is quasimonotone. Conversely, suppose that $f$ is not quasiconvex, that is, one can find three points $a, b, c \in X$ with $b \in(a, c)$ such that $f(b)>\max \{f(a), f(c)\}$. In view of Lemma 5.1, there exists $x \in[a, b]$ and $y \in[c, b]$ such that $f_{-}^{\prime}(x, b-a)>0$, and $f_{-}^{\prime}(y, a-b)>0$. It is evident that $y-x=\alpha(b-a)$ and $x-y=\beta(b-a)$ for some positive numbers $\alpha$ and $\beta$. Since the bifunction $f_{-}^{\prime}$ is positively homogenuous in the second variable, we have

$$
\begin{aligned}
& f_{-}^{\prime}(x, y-x)=\alpha f_{-}^{\prime}(x, b-a)>0 \\
& f_{-}^{\prime}(y, x-y)=\beta f_{-}^{\prime}(y, b-c)>0 .
\end{aligned}
$$

This shows that the bifunction $f_{-}^{\prime}$ is not quasimonotone. For the directional upper derivatives the proof is similar.

It is to be noted that several existing characterisations of quasiconvex functions established in $[1,2,4,5,6,7,10,11,15]$ can be obtained as direct consequences from Theorem 3.2 and Theorem 5.2. As an illustration let us derive two relevant results: the first one is Schaible's concerning quadratic functions, the second one is Crouzeix's on 
second order conditions of quasiconvexity. Let $f$ be a quadratic function of the form

$$
f(x)=\frac{1}{2} x A x+b x
$$

where $A$ is a symmetric $n \times n$ matrix, $b$ is an $n$-vector. It is known that by an appropriate affine transform $f(x)$ can be written in a canonical form:

$$
f(x)=-\frac{1}{2} \sum_{i=1}^{k} x_{i}^{2}+\frac{1}{2} \sum_{i=k+1}^{r} x_{i}^{2}+\gamma x_{r+1}+c
$$

where $0 \leqslant k \leqslant r \leqslant n, \gamma$ is either 0 or $1, c$ is a constant and $x=\left(x_{1}, \ldots, x_{n}\right)$.

COROllary 5.3. [15] Assume that the quadratic function $f(x)$ of the form (13) is not convex. Then it is quasiconvex on a convex set $C$ with a nonempty interior if and only if $k=1, \gamma=0$ and $C$ is contained either in $\left\{x \in \mathbb{R}^{n}: q(x) \leqslant 0, x_{1} \geqslant 0\right\}$ or in $\left\{x \in \mathbb{R}^{n}: q(x) \leqslant 0, x_{1} \leqslant 0\right\}$, where $q(x)=-x_{1}^{2} / 2+\left(x_{2}^{2}+\ldots+x_{r}^{2}\right) / 2$.

PROOF: It is obvious that $f(x)$ is not convex if and only if $k \geqslant 1$. Moreover, the gradient of $f$ is $\nabla f(x)=\left(-x_{1}, \ldots,-x_{k}, x_{k+1}, \ldots, x_{r}, \gamma, 0, \ldots, 0\right)$. Hence, $f^{\prime}(x, v)=$ $\langle\nabla f(x), v\rangle$ for every $x, v \in \mathbb{R}^{n}$.

Assume $k \geqslant 2$, and take any point $a=\left(a_{1}, \ldots, a_{n}\right) \in$ int $C$ with $a_{1} \neq 0$. Let $a^{\prime}=\left(-a_{2}, a_{1}, 0, \ldots, 0\right)$ and $x=a+\varepsilon a^{\prime}, y=a-\varepsilon a^{\prime}$, where $\varepsilon$ is small enough so that both $x, y$ belong to $C$. It is evident that $f^{\prime}(x, y-x)=f^{\prime}(y, x-y)=2 \varepsilon^{2}\left(a_{1}^{2}+a_{2}^{2}\right)>0$. In view of Theorem 5.1, $f$ is not quasiconvex on $C$.

Assume further that $\gamma=1$ and as before take any point $a \in$ int $C$. Let $y=$ $\left(y_{1}, \ldots, y_{n}\right)$ with $y_{i}=x_{i}$, for $i \neq 1, r+1$, and $y_{1}=x_{1}+\varepsilon, y_{r+1}=x_{r+1}+\varepsilon x_{1}+\varepsilon^{2} / 2$, where $\varepsilon$ is so small that $y$ belongs to $C$. Again one has $f^{\prime}(a, b-a)=f^{\prime}(b, a-b)=\varepsilon^{2} / 2>0$ and $f$ cannot be quasiconvex on $C$.

Thus, let us now consider the case $k=1$ and $\gamma=0$. We show that if $C$ contains a point of positive value of $q(x)$, then $f$ is not quasiconvex on $C$. In fact, let $a \in$ int $C$ with $q(a)>0$. If $a_{1}=0$, take $x=\left(\varepsilon, a_{2}, \ldots, a_{n}\right), y=\left(-\varepsilon, a_{2}, \ldots, a_{n}\right)$ where $\varepsilon$ is positive and so small that $x$ and $y$ belong to $C$. If $a_{1} \neq 0$, take $x=a$ and $y=\left(a_{1}+\alpha a_{1}, a_{2}+(\beta+\varepsilon) a_{2}, \ldots, a_{n}+(\beta+\varepsilon) a_{n}\right)$ with $\alpha=\sum_{i=2}^{r} a_{i}^{2} / k, \beta=a_{1}^{2} / k$, where $k$ and $\varepsilon>0$ are chosen so that $x, y \in C$ and $\alpha^{2} a_{1}^{2}-\left(\beta^{2}+\varepsilon(\beta+1+\varepsilon)\right) \sum_{i=2}^{r} a_{i}^{2}>0$. The existence of $k$ is evident. The existence of $\varepsilon$ follows from the fact that $q(a)>0$, hence $\alpha^{2} a_{1}^{2}-\beta^{2} \sum_{i=2}^{r} a_{i}^{2}>0$. A direct calculation shows that in both cases $f^{\prime}(x, y-x)$ and $f^{\prime}(y, x-y)$ are positive, hence $f$ cannot be quasiconvex.

Furthermore, if a point $x$ with the first component $x_{1}$ nonzero and its first componentwise opposite point $\bar{x}=\left(-x_{1}, x_{2}, \ldots, x_{n}\right)$ both are in $C$, then the middle point 
$a=\left(0, x_{2}, \ldots, x_{n}\right)$ is in $C$ too. Moreover, since int $C$ is nonempty, it must contain a point $a^{\prime}=\left(0, a_{2}, \ldots, a_{n}\right)$ with $\sum_{i=2}^{r} a_{i}^{2}>0$ in its interior. At this point $q\left(a^{\prime}\right)>0$. Hence $f$ cannot be quasiconvex on $C$. In summary, the conditions given in the corollary are necessary for $f(x)$ to be quasiconvex on $C$. They are also sufficient. Indeed, let $x, y$ be any points in $C$, say $x_{1} \geqslant 0, y_{1} \geqslant 0$ and $q(y) \leqslant q(x) \leqslant 0$. Using the Cauchy-Schwarz inequality one has

$$
\begin{aligned}
\langle\nabla f(x), y-x\rangle & =-x_{1} y_{1}+\sum_{i=2}^{r} x_{i} y_{i}-2 q(x) \\
& \leqslant-x_{1} y_{1}+\left(x_{1}^{2}+2 q(x)\right)^{1 / 2}\left(y_{1}^{2}+2 q(y)\right)^{1 / 2}-2 q(x) \\
& \leqslant-x_{1} y_{1}+\left(x_{1} y_{1}+2 q(x)\right)-2 q(x)=0 .
\end{aligned}
$$

By Theorem 5.2, $f$ is quasiconvex on $C$.

Let us remark that the condition $k=1, \gamma=0$ amounts to saying that $\operatorname{rank}(A, b)=\operatorname{rank} A$ and $A$ has exactly one negative eigenvalue.

COROLlaRY 5.4. $[4,16]$ Let $f$ be a real twice differentiable function on an open set $C \subseteq \mathbb{R}^{n}$. Then $f$ is quasiconvex on $C$ if and only if $x \in C$ and $\langle\nabla f(x), v\rangle=0$ implies either $\left\langle\nabla^{2} f(x) v, v\right\rangle>0$ or $\left\langle\nabla^{2} f(x) v, v\right\rangle=0$ and the function $f(x+t v)$ is quasiconvex in $t$ with $x+t v \in C$.

Proof: Assume that $f$ is quasiconvex and let $x \in C$ with $\langle\nabla f(x), v\rangle=0$ for some $v \in \mathbb{R}^{n}$. If $\left\langle\nabla^{2} f(x) v, v\right\rangle<0$, one takes $a=x-\varepsilon v, b=x+\varepsilon v$ with $\varepsilon$ small enough. Then $f^{\prime}(a, b-a)>0$ and $f^{\prime}(b, a-b)>0$, which by Theorem 5.2 is impossible. Hence $\left\langle\nabla^{2} f(x) v, v\right\rangle \geqslant 0$. It is clear that $f(x+t v)$ is quasiconvex in $t$. Conversely, suppose that the condition of the corollary is fulfilled but $f(x)$ is not quasiconvex on $C$. In view of Theorem 5.2, there exist $a, b \in C$ such that $f^{\prime}(a, b-a)>0$ and $f^{\prime}(b, a-b)>0$. Let $x \in(a, b)$ be a point maximising $f$ on $[a, b]$. Then $f^{\prime}(x, v)=0$ where $v=b-a$ and by the condition of the corollary, $\left\langle\nabla^{2} f(x) v, v\right\rangle$ must be positive. This is impossible because $x$ is a maximum of $f$ on $[a, b]$, and the proof is complete.

We finish this section with the remark that unlike the convex case the directional upper derivative and the directional lower derivative of a quasiconvex function do not coincide in general. For instance the function

$$
f(x)= \begin{cases}0 & \text { if } x \leqslant 0 \\ x^{2} \sin \frac{1}{x}-3 x & \text { if } 0<x<1 \\ \sin 1-3 & \text { otherwise, }\end{cases}
$$

is quasiconvex on $\mathbb{R}$ but $f_{+}^{\prime}(0,1)=-2$ and $f_{-}^{\prime}(0,1)=-4$. Moreover, as in the case of convex functions $[9,8]$, without the lower semicontinuity of $f(x)$ the results presented 
in our paper are not true. The function

$$
f(x)= \begin{cases}0 & \text { if } x \neq 0 \\ 1 & \text { otherwise }\end{cases}
$$

is not quasiconvex on $\mathbb{R}$, neverthless its generalised subgradient as well as directional derivative are monotone (and hence quasimonotone).

\section{REFERENCES}

[1] M. Avriel, Nonlinear programming: analysis and methods (Prentice-Hall, Englewood Cliffs, N.J., 1976).

[2] K.J. Arrow and A.C. Enthoven, 'Quasiconcave programming', Econometrica 29 (1961), 779-800.

[3] F.H. Clarke, Optimization and nonsmooth analysis (Wiley, New York, 1983).

[4] J.-P Crouzei, Contributions à l'étude des fonctions quasiconvexes, Ph.D. Dissertation (Université de Clermont, France, 1977).

[5] W.E. Diewert, M. Avriel and I. Zang, 'Nine kinds of quasiconcavity and concavity', J. Econom. Theory (1981).

[6] B. de Finetti, 'Sulle stratificazioni convesse', Ann. Mat. Pura Appl. 30 (1949), 173-183.

[7] R. Ellaia and A. Hassouni, 'Characterizations of nonsmooth functions through their generalized gradients', Optimization 22 (1991), 401-416.

[8] D.T. Luc, 'On the maximal monotonicity of subdifferentials', Acta Math. Veitnam 18 (1993), 99-106.

[9] D.T. Luc and S. Swaminathan, 'A characterization of convex functions', J. Nonlinear Analysis, Theory, Methods and Appl. 20 (1993), 697-701.

[10] O.L. Mangasarian, Nonlinear programming (McGraw-Hill Book Co., New York, 1969).

[11] J. Ponstein, 'Seven kinds of convexity', SIAM Rev. 9 (1967), 115-119.

[12] J.P. Penot and P.H. Quang, 'On quasimonotone maps', private communication.

[13] R.T. Rockafellar, 'Generalized dirivatives and subgradients of nonconvex functions', Canad. J. Math. XXXII (1980), 257-280.

[14] R.T. Rockafellar, The theory of subgradients and its applications to problems of optimization. Convex and nonconvex functions (Heldermann-Verlag, Berlin, 1981).

[15] S. Schaible, 'Generalized convexity of quadratic functions', in Generalized concavity in optimization and economics, (S. Schaible and W.T. Ziemba, Editors) (Academic Press, New York, 1981), pp. 183-197.

[16] S. Schaible and W.T. Ziemba, Generalized concavity in optimization and economics (Academic Press, New York, 1981).

[17] D. Zagrodny, 'Approximate mean value theorem for upper subderivatives', J. Nonlinear Analysis, Theory, Methods and Appl. 12 (1988), 1413-1428. 
Institute of Mathematics

PO Box 631, Boho

10000 Hanoi

Vietnam 